\title{
Global Stability and Bifurcations of a Diffusive Ratio-Dependent Holling-Tanner System
}

\author{
Wenjie Zuo \\ College of Science, China University of Petroleum (East China), Qingdao, Shandong 266580, China \\ Correspondence should be addressed to Wenjie Zuo; zuowjmail@163.com
}

Received 8 May 2013; Accepted 17 July 2013

Academic Editor: Chun-Lei Tang

Copyright (C) 2013 Wenjie Zuo. This is an open access article distributed under the Creative Commons Attribution License, which permits unrestricted use, distribution, and reproduction in any medium, provided the original work is properly cited.

\begin{abstract}
The dynamics of a diffusive ratio-dependent Holling-Tanner predator-prey system subject to Neumann boundary conditions are considered. By choosing the ratio of intrinsic growth rates of predators to preys as a bifurcation parameter, the existence and stability of spatially homogeneous and nonhomogeneous Hopf bifurcations and steady state bifurcation are investigated in detail. Meanwhile, we show that Turing instability takes place at a certain critical value; that is, the stationary solution becomes unstable induced by diffusion. Particularly, the sufficient conditions of the global stability of the positive constant coexistence are given by the upper-lower solutions method.
\end{abstract}

\section{Introduction}

Predator-prey systems describing the interaction between two species with positive-negative feedbacks continue to arouse the interest of applied mathematicians and ecologists. The functional response is the important component depicting the predator-prey relationship [1]. In order to better characterize the ecological interaction such as lynx and hare and sparrow and sparrow hawk especially by Tanner [2] and Wollkind et al. [3], May developed the so-called Holling-Tanner predator-prey model [4] which takes the following form:

$$
\begin{gathered}
\frac{d U}{d T}=r U(T)\left(1-\frac{U(T)}{K}\right)-\frac{m U(T) V(T)}{u(T)+q}, \\
\frac{d V}{d T}=s V(T)\left(1-h \frac{V(T)}{U(T)}\right),
\end{gathered}
$$

where $U(T), V(T)$ stand for the prey and the predator densities, respectively. And in ecological senses, $r, K, m$, $q, s, h$ are positive constants which denote prey intrinsic growth rate, carrying capacity, capturing rate, half capturing saturation constant, predator intrinsic growth rate, and conversion rate of prey into predator, respectively. The functional response $m U /(U+q)$ is of Michaelis-Menten type in enzyme-substrate kinetics, proposed by Holling [5].

Hsu and Huang [6] proved the global stability of the positive equilibrium of System (1) by applying the Dulac's criterion and constructing Lyapunov function. Gasull et al. [7] showed that the stable positive equilibrium is surrounded by two limit cycles. Thus, the local stability does not imply the global stability.

Spatial diffusion is ubiquitous. Assuming the preys and the predators are in an isolate patch, I neglect the impact of migration, including immigration and emigration, and only consider the diffusion of the spatial domain. It has been shown that the diffusion can generate the more rich spatiotemporal complex [8-10]. A diffusive Holling-Tanner predator-prey system is as follows:

$$
\begin{gathered}
\frac{\partial U}{\partial T}=D_{1} U_{x x}+r U(x, T)\left(1-\frac{U(x, T)}{K}\right) \\
-\frac{m U(x, T) V(x, T)}{U(x, T)+q}, \quad x \in(0, l \pi), T>0, \\
\frac{\partial V}{\partial T}=D_{2} V_{x x}+s V(x, T)\left(1-h \frac{V(x, T)}{U(x, T)}\right), \\
x \in(0, l \pi), T>0, \\
U_{x}(0, T)=U_{x}(l \pi, T)=V_{x}(0, T)=V_{x}(l \pi, T)=0, \quad T \geq 0, \\
U(x, 0)=U_{0}(x) \geq 0, \quad V(x, 0)=V_{0}(x) \geq 0, \quad x \in[0, l \pi] .
\end{gathered}
$$


Ma and Li [11] investigated the existence of Hopf bifurcation and the steady state bifurcations of simple and double eigenvalues of System (2). Peng and Wang [12] proved the global stability of the unique positive equilibrium of System (2) under certain hypothesis by constructing Lyapunov function. Chen and Shi [13] improved the results given in [12].

Some biologists have questioned the functional response solely depending on the prey density [14-16], especially when predators have to search for food, (and therefore have to share or compete for food). Numerous fields and laboratory experiments have revealed that a more reasonable functional response should be the so-called ratio-dependent predatorprey theory $[14,15]$, which can be strongly stated as that the per capita predator growth rate should be the function of the ratio of predator abundance. A predator-prey system with the ratio-dependent functional response can exhibit rich dynamics $[17,18]$.

In this paper, we consider the following diffusive ratiodependent Holling-Tanner predator-prey system:

$$
\begin{gathered}
\frac{\partial U}{\partial T}=D_{1} U_{x x}+r U(x, T)\left(1-\frac{U(x, T)}{K}\right) \\
-\frac{m U(x, T) V(x, T)}{U(x, T)+q V(x, T)}, \quad x \in(0, l \pi), T>0, \\
\frac{\partial V}{\partial T}=D_{2} V_{x x}+s V(x, T)\left(1-h \frac{V(x, T)}{U(x, T)}\right), \\
x \in(0, l \pi), T>0, \\
U_{x}(0, T)=U_{x}(l \pi, T)=V_{x}(0, T)=V_{x}(l \pi, T)=0, \quad T \geq 0, \\
U(x, 0)=U_{0}(x) \geq 0, \quad V(x, 0)=V_{0}(x) \geq 0, \quad x \in[0, l \pi] .
\end{gathered}
$$

M. Banerjee and S. Banerjee [19] examined the existence of Turing and non-Turing patterns of System (3) by numerical simulations and revealed the fact that Hopf bifurcation is essential for spatiotemporal chaos. Liang and Pan [20] established the global stability of positive equilibrium of System (3) without diffusion by constructing Lyapunov function and obtained the uniqueness of the limit cycle.

My work is the extension of [11, 19]. In addition, what is more important in ecosystem is whether the species would coexist in the long run; that is, whether the positive equilibrium is globally asymptotically stable. In this paper, we aim to study Hopf bifurcations and steady state bifurcations induced by intrinsic growth rate and Turing instability induced by diffusion. Particularly, the global stability of the unique positive equilibrium is obtained by the upper-lower solutions method under certain conditions. Simulations are carried out to strongly support the theoretical results.

The rest of the paper is organized as follows. In Section 2, the instability of the semitrivial equilibrium and the local stability of the positive coexistence of system (3) are studied by the distribution of eigenvalues. And the existence, stability, and direction of Hopf bifurcations are investigated in detail as the intrinsic growth rate $\lambda$ crosses the critical value. Turing bifurcation induced by diffusion takes place at the certain critical values. In Section 3, the steady state bifurcations are considered with $\lambda$ as the bifurcation parameter and the interaction of Hopf bifurcation and steady state bifurcation are discussed. In Section 4, the global stability of the unique positive coexistence is proved by the upper-lower solutions method.

\section{Hopf Bifurcations and Turing Bifurcations}

For simplicity of later discussion, we suppose that $D_{1}=r$. Introducing the following dimensionless variables:

$$
u=\frac{U}{K}, \quad v=\frac{m V}{r K}, \quad t=r T
$$

System (3) can be transformed into

$$
\begin{gathered}
\frac{\partial u}{\partial t}=u_{x x}+u(x, t)(1-u(x, t)) \\
-\frac{u(x, t) v(x, t)}{u(x, t)+\alpha v(x, t)}, \quad x \in(0, l \pi), t>0, \\
\frac{\partial v}{\partial t}=d v_{x x}+\lambda v(x, t)\left(\beta-\frac{v(x, t)}{u(x, t)}\right), \\
x \in(0, l \pi), t>0, \\
u(x, 0)=u_{0}(x) \geq 0, \quad v(x, 0)=v_{0}(x) \geq 0, \quad x \in[0, l \pi],
\end{gathered}
$$

where $d=D_{2} / D_{1}, \alpha=q r / m, \lambda=s h / m, \beta=m / h r$.

It is easy to check that System (5) has always a boundary equilibrium $E_{0}(1,0)$ and has a positive constant coexistence $E\left(u^{*}, v^{*}\right)$ if and only if $\alpha \beta+1>\beta$, where

$$
u^{*}=1-\frac{\beta}{\alpha \beta+1}>0, \quad v^{*}=\beta u^{*} .
$$

The characteristic equations of the linearization of (5) at $E_{0}(1,0)$ are given by

$$
\left(\mu+\frac{n^{2}}{l^{2}}+1\right)\left(\mu+d \frac{n^{2}}{l^{2}}-\lambda \beta\right)=0, \quad n=0,1,2, \ldots
$$

Thus,

$$
\mu_{1}=-\frac{n^{2}}{l^{2}}-1, \quad \mu_{2}=\lambda \beta-d \frac{n^{2}}{l^{2}}, \quad n=0,1, \ldots
$$

Hence, $E_{0}(1,0)$ is always unstable since (7) has at least a positive root $\mu=\lambda \beta$. Next we study the local stability of the positive steady state $E\left(u^{*}, v^{*}\right)$ and the existence of bifurcations. 
2.1. Stability of the Positive Coexistence and Hopf Bifurcations. The linearized operator of System (5) at $E\left(u^{*}, v^{*}\right)$ is as follows:

$$
\begin{gathered}
L(\lambda)=\left(\begin{array}{cc}
\frac{\partial^{2}}{\partial x^{2}}+\frac{\alpha \beta^{2}+2 \beta}{(\alpha \beta+1)^{2}}-1 & -\frac{1}{(\alpha \beta+1)^{2}} \\
\lambda \beta^{2} & d \frac{\partial^{2}}{\partial x^{2}}-\lambda \beta
\end{array}\right), \\
L_{n}(\lambda)=\left(\begin{array}{cc}
-\frac{n^{2}}{l^{2}}+\frac{\alpha \beta^{2}+2 \beta}{(\alpha \beta+1)^{2}}-1 & -\frac{1}{(\alpha \beta+1)^{2}} \\
\lambda \beta^{2} & -d \frac{n^{2}}{l^{2}}-\lambda \beta
\end{array}\right) .
\end{gathered}
$$

The characteristic equations corresponding to $L_{n}(\lambda)$ are

$$
\mu^{2}-T_{n}(\lambda) \mu+D_{n}(\lambda)=0, \quad n=0,1,2, \ldots
$$

where

$$
\begin{aligned}
T_{n}(\lambda)= & -\frac{(1+d) n^{2}}{l^{2}}-\lambda \beta+\frac{\alpha \beta^{2}+2 \beta}{(\alpha \beta+1)^{2}}-1 \\
= & -\frac{(1+d) n^{2}}{l^{2}}-\left(\lambda-\lambda_{0}^{H}\right) \beta, \\
D_{n}(\lambda)= & \frac{d n^{4}}{l^{4}}+\frac{n^{2}}{l^{2}}\left(d\left(1-\frac{\alpha \beta^{2}+2 \beta}{(\alpha \beta+1)^{2}}\right)+\lambda \beta\right) \\
& +\lambda \beta\left(1-\frac{\beta}{1+\alpha \beta}\right) \\
= & \frac{d n^{4}}{l^{4}}+\frac{n^{2}}{l^{2}}\left(\lambda-d \lambda_{0}^{H}\right) \beta+\lambda \beta u^{*},
\end{aligned}
$$

where $\lambda_{0}^{H}=(1 / \beta)\left(\left(\alpha \beta^{2}+2 \beta\right) /(\alpha \beta+1)^{2}-1\right)$.

Obviously, if $\alpha \beta^{2}+2 \beta \leq(\alpha \beta+1)^{2}$, then $T_{n}(\lambda)<0, D_{n}(\lambda)>$ 0 , which implies that all the roots of (10) are negative realpart. Thus the unique positive constant equilibrium $E\left(u^{*}, v^{*}\right)$ is locally asymptotically stable.

Indeed, in Section 4, we can prove the global stability of the unique positive coexistence under the stronger conditions. Next, we will seek all the possible Hopf bifurcation values $\lambda_{0}$, so we assume $\alpha \beta^{2}+2 \beta>(1+\alpha \beta)^{2}$ holds.

We will identify Hopf bifurcation value $\lambda_{0}$ which satisfies

$$
\begin{gathered}
T_{n}\left(\lambda_{0}\right)=0, \quad D_{n}\left(\lambda_{0}\right)>0, \\
T_{j}\left(\lambda_{0}\right) \neq 0, \quad D_{j}\left(\lambda_{0}\right) \neq 0, \quad \forall j \neq n .
\end{gathered}
$$

From (11), $T_{n}(\lambda)<0$, for all $\lambda \in\left(\lambda_{0}^{H},+\infty\right)$, which means that the possible Hopf bifurcation points belong to the interval $\left(0, \lambda_{0}^{H}\right]$. For any Hopf bifurcation point $\lambda^{*} \in\left(0, \lambda_{0}^{H}\right], \alpha(\lambda) \pm$ $i \omega(\lambda)$ are a pair of conjugate eigenvalues of $L_{n}(\lambda)$ near $\lambda=\lambda^{*}$, where

$$
\alpha(\lambda)=\frac{T_{n}(\lambda)}{2}, \quad \omega(\lambda)=\frac{1}{2} \sqrt{4 D_{n}(\lambda)-T_{n}^{2}(\lambda)},
$$

and $\alpha^{\prime}\left(\lambda^{*}\right)=-\beta / 2<0$.
Hence, all Hopf bifurcation points reduce to the following set:

$$
\begin{aligned}
\Lambda_{1}=\left\{\lambda \in\left(0, \lambda_{0}^{H}\right], \text { for some } n \in N,\right. \\
\text { the condition (12) is satisfied }\} .
\end{aligned}
$$

Obviously, $T_{n}(\lambda)=0$ is equivalent to

$$
\begin{gathered}
\lambda_{n}=\lambda_{0}^{H}-\frac{(1+d) n^{2}}{\beta l^{2}}, \\
D_{n}\left(\lambda_{n}\right)=-\frac{n^{4}}{l^{4}}-\frac{n^{2}}{l^{2}} B_{0}+\lambda_{0}^{H} \beta u^{*},
\end{gathered}
$$

where $B_{0}=2-\left(2 \alpha \beta^{2}+3 \beta-d \beta\right) /(1+\alpha \beta)^{2}$. Since $D_{0}\left(\lambda_{0}\right)=$ $\lambda_{0}^{H} \beta u^{*}>0$, there exists $N_{0} \in N^{+}$such that $D_{n}\left(\lambda_{n}\right)>0$, $n=0,1,2, \ldots, N_{0}$, and $D_{n}\left(\lambda_{n}\right) \leq 0, n=N_{0}+1, N_{0}+2, \ldots$

Clearly, $T_{i}\left(\lambda_{n}\right)=\left((1+d) / l^{2}\right)\left(n^{2}-i^{2}\right) \neq 0$, for all $i \neq n$. And for $i \neq n$,

$$
D_{i}\left(\lambda_{n}\right)=\frac{d i^{4}}{l^{4}}+\frac{i^{2}}{l^{2}}\left(\lambda_{n}-d \lambda_{0}^{H}\right) \beta+\lambda_{n} \beta u^{*} .
$$

We discuss $D_{i}\left(\lambda_{n}\right)>0$, for all $i \neq n$ in two cases.

Case 1. We can choose small enough $d>0$ such that $\lambda_{n}-$ $d \lambda_{0}^{H}>0$, that is, for $n=0,1, \ldots, N_{0}, d<d_{0}$, where

$$
d_{0}:=\frac{\beta l^{2} \lambda_{0}^{H}-N_{0}^{2}}{\beta l^{2} \lambda_{0}^{H}+N_{0}^{2}}>0 .
$$

Case 2. When $d>d_{0}$,

$$
\begin{gathered}
d \lambda_{0}^{H}-\lambda_{n}=(d-1) \lambda_{0}^{H}+\frac{(1+d) n^{2}}{l^{2} \beta} \\
\leq(d-1) \lambda_{0}^{H}+\frac{(1+d) N_{0}^{2}}{l^{2} \beta} \triangleq M^{*}, \\
\lambda_{n} u^{*} \geq\left(\lambda_{0}^{H}-\frac{(1+d) N_{0}^{2}}{l^{2} \beta}\right) u^{*}=\left(d \lambda_{0}^{H}-M^{*}\right) u^{*} .
\end{gathered}
$$

Therefore,

$$
D_{i}\left(\lambda_{n}\right) \geq \frac{d i^{4}}{l^{4}}-\frac{i^{2}}{l^{2}} M^{*} \beta+\left(d \lambda_{0}^{H}-M^{*}\right) u^{*} \beta=g\left(\frac{i^{2}}{l^{2}}\right) .
$$

In order that $g(y)=d y^{2}-M^{*} \beta y+\left(d \lambda_{0}^{H}-M^{*}\right) u^{*} \beta>0$ for all $y \in R$, we only need $\left(M^{*}\right)^{2} \beta<4 d\left(d \lambda_{0}^{H}-M^{*}\right) u^{*}$. Thus, $D_{i}\left(\lambda_{n}\right)>0$.

Clearly, $\lambda_{0}^{H}=\max _{n \in\left\{0,1, \ldots, N_{0}\right\}} \lambda_{n}$.

Summarizing the above analysis results, we draw our main conclusion in this subsection.

Theorem 1. Suppose $\alpha, \beta, d>0$ and $\alpha \beta+1>\beta, \alpha \beta^{2}+2 \beta>$ $(1+\alpha \beta)^{2}$ hold. If there exists $d_{0}>0$ such that $0<d<d_{0}$, or $\left(M^{*}\right)^{2} \beta<4 d\left(d \lambda_{0}^{H}-M^{*}\right) u^{*}$, where $d_{0}$ and $M^{*}$ are defined 
by (18) and (19), respectively. Then for System (5), the following results are true:

(1) The positive constant steady state $E\left(u^{*}, v^{*}\right)$ is locally asymptotically stable for $\lambda \in\left(\lambda_{0}^{H},+\infty\right)$ and unstable for $\lambda \in\left(0, \lambda_{0}^{H}\right)$;

(2) System (5) undergoes a Hopf bifurcation at $\lambda=\lambda_{n}, n=$ $0,1, \ldots, N_{0}$ (defined by (15)) and the bifurcating periodic solutions can be parameterized into the following forms:

$$
\begin{aligned}
& u(s)(x, t)=s\left(a_{n} e^{2 \pi i t / T(s)}+\overline{a_{n}} e^{-2 \pi i t / T(s)}\right) \cos \frac{n x}{l}+o(s) \\
& v(s)(x, t)=s\left(b_{n} e^{2 \pi i t / T(s)}+\overline{b_{n}} e^{-2 \pi i t / T(s)}\right) \cos \frac{n x}{l}+o(s)
\end{aligned}
$$

Furthermore, the periodic solutions bifurcating from $E\left(u^{*}, v^{*}\right)$ at $\lambda=\lambda_{0}^{H}$ are spatially homogeneous. And bifurcating periodic solutions from $\lambda=\lambda_{n},\left(n=1, \ldots, N_{0}\right)$ are spatially inhomogeneous.

Next we only consider the direction and stability of Hopf bifurcation at $\lambda=\lambda_{0}^{H}$.

Theorem 2. For the System (5), the Hopf bifurcations at $\lambda=$ $\lambda_{0}^{H}$ are backward, and the bifurcating periodic solutions are locally asymptotically stable.

Proof. Here we apply the notations and calculations in $[9,21]$, we put

$$
\begin{gathered}
q:=\left(\begin{array}{l}
a_{0} \\
b_{0}
\end{array}\right)=\left(\begin{array}{c}
\frac{\lambda_{0}^{H} \beta+i \omega_{0}}{\lambda_{0}^{H} \beta^{2}} \\
1
\end{array}\right), \\
q^{*}:=\left(\begin{array}{l}
a_{0}^{*} \\
b_{0}^{*}
\end{array}\right)=\left(\begin{array}{c}
\frac{\lambda_{0}^{H} \beta^{2}}{2 l \pi \omega_{0}} i \\
\frac{\omega_{0}-i \lambda_{0}^{H} \beta}{2 l \pi \omega_{0}}
\end{array}\right),
\end{gathered}
$$

such that $\left\langle q^{*}, q\right\rangle=1$ and $\left\langle q^{*}, \bar{q}\right\rangle=0$, where $\omega_{0}=\sqrt{\lambda_{0}^{H} \beta u^{*}}$.

Recall that in our context,

$$
\begin{gathered}
f(\lambda, u, v)=u(1-u)-\frac{u v}{u+\alpha v}, \\
g(\lambda, u, v)=\lambda v\left(\beta-\frac{v}{u}\right) .
\end{gathered}
$$

By direct computation, it follows that

$$
\begin{aligned}
& c_{0}=-\frac{2}{\beta^{2}}+\frac{2 \omega_{0}^{2}}{\left(\lambda_{0}^{H}\right)^{2} \beta^{4}}-\frac{2 \alpha}{(\alpha \beta+1)^{3} \lambda_{0}^{H} \beta}-\frac{4 \omega_{0}}{\lambda_{0}^{H} \beta^{3}} i, \\
& d_{0}=\frac{2}{\beta},
\end{aligned}
$$

$$
\begin{aligned}
& e_{0}=-\frac{2}{\beta^{2}}-\frac{2 u^{*}}{\lambda_{0}^{H} \beta^{3}}+\frac{2 \alpha}{(\alpha \beta+1)^{3} \lambda_{0}^{H} \beta}, \quad f_{0}=-\frac{2}{\beta}, \\
& g_{0}=\frac{\alpha}{\lambda_{0}^{H} \beta^{2} u^{*}(\alpha \beta+1)^{4}}\left(-2 \alpha \beta-2-\frac{3 \omega_{0}}{\lambda_{0}^{H} \beta} i\right), \\
& h_{0}=\frac{2}{u^{*}}\left(\frac{1}{\beta^{2}}+\frac{3 \omega_{0}}{\lambda_{0}^{H} \beta^{3}} i\right),
\end{aligned}
$$

and

$$
\begin{aligned}
&\left\langle q^{*}, Q_{q q}\right\rangle=-\frac{1}{\beta}+i\left(\frac{2 \lambda_{0}^{H}}{\omega_{0}}-\frac{\omega_{0}}{\lambda_{0}^{H} \beta^{2}}+\frac{\alpha \beta}{(\alpha \beta+1)^{3} \omega_{0}}\right), \\
&\left\langle q^{*}, Q_{q \bar{q}}\right\rangle=\overline{\left\langle\overline{q^{*}}, Q_{q \bar{q}}\right\rangle}=-\frac{1}{\beta}+\frac{i}{\omega_{0}}\left(\frac{u^{*}}{\beta}-\frac{\alpha \beta}{(\alpha \beta+1)^{3}}\right), \\
&\left\langle\overline{q^{*}}, Q_{q q}\right\rangle= \frac{3}{\beta}-i\left(\frac{2 \lambda_{0}^{H}}{\omega_{0}}-\frac{\omega_{0}}{\lambda_{0}^{H} \beta^{2}}+\frac{\alpha \beta}{(\alpha \beta+1)^{3} \omega_{0}}\right), \\
&\left\langle q^{*}, C_{q q \bar{q}}\right\rangle=-\left(\frac{3 \alpha}{2 u^{*} \lambda_{0}^{H} \beta(\alpha \beta+1)^{4}}+\frac{2}{\beta^{2} u^{*}}\right) \\
&+i\left(\frac{\alpha}{\omega_{0} u^{*}(\alpha \beta+1)^{3}}+\frac{\lambda_{0}^{H}}{\omega_{0} u^{*} \beta}+\frac{3 \omega_{0}}{\lambda_{0}^{H} \beta^{3} u^{*}}\right) .
\end{aligned}
$$

Hence, it is direct to calculate, by (24) and (25),

$$
\begin{aligned}
H_{20}= & \left(\begin{array}{c}
c_{0} \\
d_{0}
\end{array}\right)-\left\langle q^{*}, Q_{q q}\right\rangle\left(\begin{array}{l}
a_{0} \\
b_{0}
\end{array}\right) \\
& -\left\langle\overline{q^{*}}, Q_{q q}\right\rangle\left(\frac{\overline{a_{0}}}{\overline{b_{0}}}\right)=\left(\begin{array}{l}
0 \\
0
\end{array}\right), \\
H_{11}= & \left(\begin{array}{c}
e_{0} \\
f_{0}
\end{array}\right)-\left\langle q^{*}, Q_{q \bar{q}}\right\rangle\left(\begin{array}{l}
a_{0} \\
b_{0}
\end{array}\right) \\
& -\left\langle\overline{q^{*}}, Q_{q \bar{q}}\right\rangle\left(\frac{\bar{a}_{0}}{b_{0}}\right)=\left(\begin{array}{l}
0 \\
0
\end{array}\right),
\end{aligned}
$$

which implies that $W_{20}=W_{11}=0$. Thus, $\left\langle q^{*}, Q_{W_{11} q}\right\rangle=$ $\left\langle q^{*}, Q_{W_{20} \bar{q}}\right\rangle=0$. Therefore, by (25) and $\omega_{0}^{2}=\lambda_{0}^{H} \beta u^{*}$, we can obtain that

$$
\begin{aligned}
\operatorname{Re} & \left(C_{1}\left(\lambda_{0}^{H}\right)\right) \\
& =\operatorname{Re}\left(\frac{i}{2 \omega_{0}}\left(g_{20} g_{11}-2\left|g_{11}\right|^{2}-\frac{1}{3}\left|g_{02}\right|^{2}\right)+\frac{g_{21}}{2}\right) \\
& =\operatorname{Re}\left(\frac{i}{2 \omega_{0}}\left(\left\langle q^{*}, Q_{q q}\right\rangle\left\langle q^{*}, Q_{q \bar{q}}\right\rangle\right)+\frac{1}{2}\left\langle q^{*}, C_{q q \bar{q}}\right\rangle\right)
\end{aligned}
$$




$$
\begin{aligned}
= & \left(\frac{u^{*}}{2 \beta^{2} \omega_{0}^{2}}-\frac{1}{2 \lambda_{0}^{H} \beta^{3}}\right)+\left(\frac{\lambda_{0}^{H}}{\beta \omega_{0}^{2}}-\frac{1}{\beta^{2} u^{*}}\right) \\
& -\frac{3 \alpha}{4 u^{*} \lambda_{0}^{H} \beta(1+\alpha \beta)^{4}} \\
= & -\frac{3 \alpha}{4 u^{*} \lambda_{0}^{H} \beta(1+\alpha \beta)^{4}}<0 .
\end{aligned}
$$

Since $\alpha^{\prime}\left(\lambda_{0}^{H}\right)=-\beta / 2$, then the bifurcating periodic solutions are backward and exist for $\tau<\tau_{0}^{H}$. And combining $T_{n}\left(\lambda_{0}^{H}\right)=$ $-\left((1+d) / l^{2}\right) n^{2}<0, D_{n}\left(\lambda_{0}^{H}\right)>0$, for all $n=1,2, \ldots, N_{0}$, we can obtain that the bifurcating periodic solutions are asymptotically stable since $\operatorname{Re}\left(C_{1}\left(\lambda_{0}^{H}\right)\right)<0$.

Next, we give an example to illustrate the theoretical results above.

Example 3. Taking $d=0.2, \alpha=0.4, \beta=1.2, l=2$, System (5) has a unique positive constant steady state $E(0.1892,0.227)$. Obviously, $\alpha \beta+1>\beta, \alpha \beta^{2}+2 \beta>(1+\alpha \beta)^{2}$. By the formula above, we obtain $\lambda_{0}^{H}=0.2989$. And $D_{0}\left(\lambda_{0}^{H}\right)<0, D_{1}\left(\lambda_{1}\right)>0$. Thus, $0<d<d_{0}=1$. By Theorems 1 and $2, E(0.1892,0.227)$ is asymptotically stable for $\lambda \in(0.2989,+\infty)$ as illustrated in Figures 1(a) and 1(b). When $\lambda$ crosses through the critical value, $E(0.1892,0.227)$ losses its stability and a family of periodic solutions appear and are asymptotically stable since $\operatorname{Re}\left(C_{1}\left(\lambda_{0}^{H}\right)\right)=-0.9215<0$ as illustrated in Figures $2(\mathrm{a})$ and 2(b).

We can compute the direction and stability of bifurcating periodic solutions by the same way as Theorem 2 and omit it here. Combining the stability theory, we have the following results.

Theorem 4. For System (5), the bifurcating periodic solutions from $\lambda=\lambda_{n}, n=1,2, \ldots, N_{0}$ (defined by (15)) are backward (forward) if $\operatorname{Re}\left(C_{1}\left(\lambda_{n}\right)\right)<(>) 0$ and the spatially inhomogeneous Hopf bifurcations are unstable.

2.2. Turing Instability Induced by Diffusion. In this subsection, we consider Turing instability with diffusion effect. As mentioned earlier, when $\alpha \beta^{2}+2 \beta \leq(\alpha \beta+1)^{2}, T_{n}(\lambda)<$ $0, D_{n}(\lambda)>0$, for all $n \in\{0,1,2, \ldots\}$. Hence, no Turing instability occurs. Next we always confine $\alpha \beta^{2}+2 \beta>(\alpha \beta+$ $1)^{2}$.

When System (5) has no diffusion, the characteristic equation $(10)$ at $E\left(u^{*}, v^{*}\right)$ can be transformed into

$$
\mu^{2}-\left(\lambda_{0}^{H}-\lambda\right) \beta \mu+\lambda \beta u^{*}=0 .
$$

Then all the roots of (28) are negative real part if $\lambda>\lambda_{0}^{H}$. Combining the stability theory [22], we easily know that the unique positive equilibrium $E\left(u^{*}, v^{*}\right)$ of System (5) without diffusion is asymptotically stable for $\lambda \in\left(\lambda_{0}^{H},+\infty\right)$ and unstable for $\lambda \in\left[0, \lambda_{0}^{H}\right)$. In the following, we mainly consider the occurrence of Turing instability induced by diffusion.
Hence we confine $\lambda \in\left(\lambda_{0}^{H},+\infty\right)$. That is, $E\left(u^{*}, v^{*}\right)$ is locally asymptotically stable without diffusion effect.

It is well-known that $E\left(u^{*}, v^{*}\right)$ is unstable if the characteristic equation (10) has at least a positive real-part root. Notice when $\lambda>\lambda_{0}^{H}, T_{n}(\lambda)<0$, for all $n=0,1,2, \ldots$. The necessary condition of the instability of the equlibirium $E\left(u^{*}, v^{*}\right)$ of System $(5)$ is $D_{n}(\lambda)<0$ for some $n \in N^{+}=\{1,2, \ldots\}$.

Recall

$$
D_{n}(\lambda)=\frac{d n^{4}}{l^{4}}+\frac{n^{2}}{l^{2}}\left(\lambda-d \lambda_{0}^{H}\right) \beta+\lambda \beta u^{*} \triangleq g\left(\frac{n^{2}}{l^{2}}\right) \text {, }
$$

where $g(x)=d x^{2}-\left(d \lambda_{0}^{H}-\lambda\right) \beta x+\lambda \beta u^{*}$.

Hence we need $\lambda \in\left(\lambda_{0}^{H}, d \lambda_{0}^{H}\right), d>1$ and $\Delta \triangleq\left(d \lambda_{0}^{H}-\right.$ $\lambda)^{2} \beta^{2}-4 d \lambda \beta u^{*}>0$. That is, $\left(d \lambda_{0}^{H}-\lambda\right) \beta>2 \sqrt{d \lambda \beta u^{*}}$. Otherwise, $D_{i}(\lambda) \geq 0$ for all $i \in N$.

Obviously, $g(x)=0$ has two positive roots $x_{1}, x_{2}$ since

$$
x_{1}+x_{2}=\left(d \lambda_{0}^{H}-\lambda\right) \beta>0, \quad x_{1} x_{2}>0,
$$

where

$$
\begin{aligned}
& x_{1}=\frac{1}{2 d}\left(\left(d \lambda_{0}^{H}-\lambda\right) \beta-\sqrt{\left(d \lambda_{0}^{H}-\lambda\right)^{2} \beta^{2}-4 d \lambda \beta u^{*}}\right), \\
& x_{2}=\frac{1}{2 d}\left(\left(d \lambda_{0}^{H}-\lambda\right) \beta+\sqrt{\left(d \lambda_{0}^{H}-\lambda\right)^{2} \beta^{2}-4 d \lambda \beta u^{*}}\right) .
\end{aligned}
$$

If we can find some $j \in N^{+}$such that $x_{1}<j^{2} / l^{2}<x_{2}$. Then $D_{j}(\lambda)<0$. That is, $\sqrt{x_{1}} l<j<\sqrt{x_{2}} l$. The constant positive coexistence $E\left(u^{*}, v^{*}\right)$ is unstable. Hence, we draw the following conclusions.

Theorem 5. Assume that $\alpha, \beta>0, d>1$, and $\alpha \beta+1>\beta$, $\alpha \beta^{2}+2 \beta>(\alpha \beta+1)^{2}$ hold. Then $E\left(u^{*}, v^{*}\right)$ is unstable for $\lambda \epsilon$ $\left(\lambda_{0}^{H}, d \lambda_{0}^{H}\right)$. That is, Turing instability occurs if

$$
\begin{gathered}
d \lambda_{0}^{H} \beta-\lambda \beta>2 \sqrt{d \lambda \beta u^{*}}, \\
\sqrt{x_{1}} l<j<\sqrt{x_{2}} l \quad \text { for some } j \in N^{+} .
\end{gathered}
$$

\section{Steady State Bifurcations}

In this section, we still choose $\lambda$ as the main bifurcation parameter with $\alpha, \beta, d$ positive constants and study the existence of the steady state bifurcation points. The steady state solutions of System (5) satisfy the following elliptic system:

$$
\begin{gathered}
u_{x x}+u(1-u)-\frac{u v}{u+\alpha v}=0, \quad x \in[0, l \pi], \\
d v_{x x}+\lambda v\left(\beta-\frac{v}{u}\right)=0, \quad x \in[0, l \pi], \\
u_{x}(0, t)=u_{x}(l \pi, t)=v_{x}(0, t) \\
=v_{x}(l \pi, t)=0, \quad t \geq 0, \\
u(x, 0)=u_{0}(x) \geq 0, \\
v(x, 0)=v_{0}(x) \geq 0 .
\end{gathered}
$$




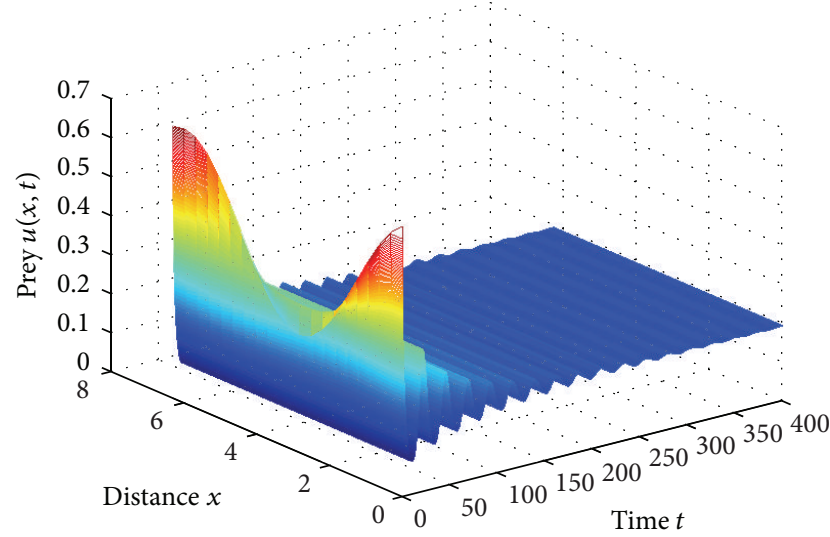

(a)

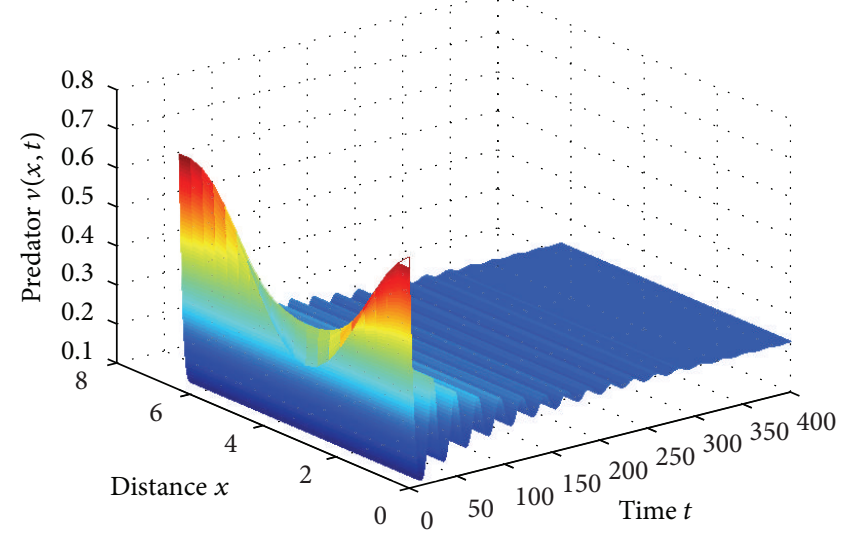

(b)

FIGURE 1: $E(0.1892,0.227)$ is asymptotically stable when $\lambda=0.32>\lambda_{0}^{H}$ and the initial function $u_{0}(x)=0.5+0.2 \cos x, v_{0}(x)=0.5+0.2 \cos x$.

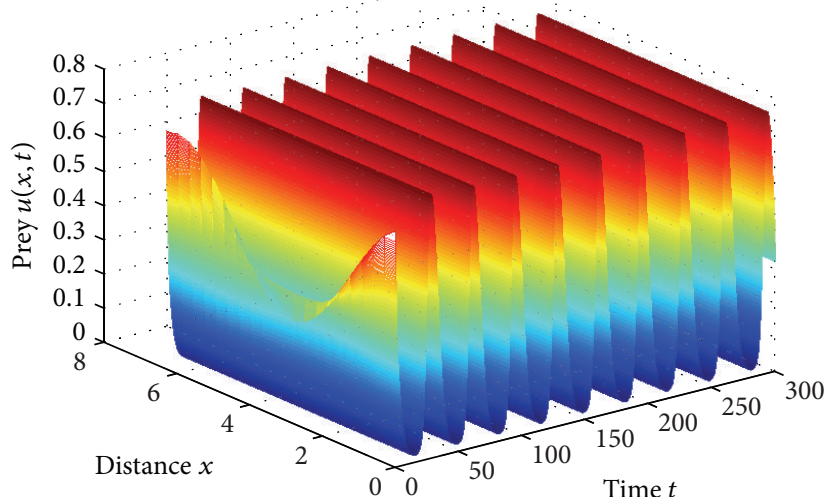

(a)

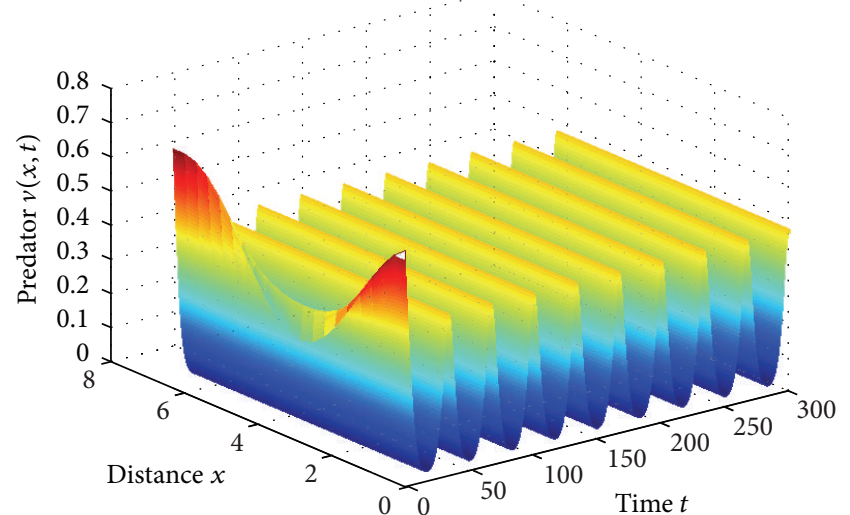

(b)

FIGURE 2: Spatially homogenous periodic solutions are asymptotically stable when $\lambda=0.24<\lambda_{0}^{H}$ and the initial function $u_{0}(x)=0.5+$ $0.2 \cos x, v_{0}(x)=0.5+0.2 \cos x$.

System (33) has a unique positive constant coexistence $E\left(u^{*}, v^{*}\right)$ if and only if $\alpha \beta+1>\beta$. By the same way as Section 2.1, the characteristic equation of the linearization of (33) at $E\left(u^{*}, v^{*}\right)$ is

$$
\mu^{2}-T_{n}(\lambda) \mu+D_{n}(\lambda)=0, \quad n=0,1,2, \ldots,
$$

where $T_{n}(\lambda), D_{n}(\lambda)$ are defined by (11).

Next we identify all the possible steady state bifurcation points by [9] which satisfy the following conditions:

$$
\begin{gathered}
D_{n}\left(\lambda_{0}\right)=0, \quad T_{n}\left(\lambda_{0}\right) \neq 0, \\
D_{m}\left(\lambda_{0}\right) \neq 0, \quad T_{m}\left(\lambda_{0}\right) \neq 0, \quad \forall m \neq n, \\
\frac{d D_{n}\left(\lambda_{0}\right)}{d \lambda} \neq 0 .
\end{gathered}
$$

For convenience, we rewrite

$$
D_{n}(\lambda)=d p^{2}+p\left(\lambda-d \lambda_{0}^{H}\right) \beta+\lambda \beta u^{*} \triangleq g(p),
$$

where $p=n^{2} / l^{2}$.
Obviously, $D_{n}(\lambda)>0$, for all $n=0,1,2, \ldots$ for $\lambda \in\left[d \lambda_{0}^{H},+\infty\right)$. Hence the potential steady state bifurcation points set is as follows:

$$
\wedge_{1}=\left\{\lambda \in\left(0, d \lambda_{0}^{H}\right) \mid(35) \text { are satisfied }\right\}
$$

with $\alpha, \beta, l, d$ fixed.

Thus $D_{n}(\lambda)=0$ has two roots as

$$
p_{ \pm}(\lambda)=\frac{1}{2 d}\left(\left(d \lambda_{0}^{H}-\lambda\right) \beta \pm \sqrt{\left(d \lambda_{0}^{H}-\lambda\right)^{2} \beta^{2}-4 d \lambda \beta u^{*}}\right) .
$$

Let

$$
\begin{aligned}
h(\lambda)= & \left(d \lambda_{0}^{H}-\lambda\right)^{2} \beta^{2}-4 d \lambda \beta u^{*} \\
= & \lambda^{2} \beta^{2}-2 d \lambda \beta\left(\lambda_{0}^{H} \beta+2 u^{*}\right) \\
& +d^{2} \beta^{2}\left(\lambda_{0}^{H}\right)^{2} .
\end{aligned}
$$


Since $\Delta \triangleq 4 d^{2} \beta^{2}\left(\lambda_{0}^{H} \beta+2 u^{*}\right)^{2}-4 \beta^{4} d^{2}\left(\lambda_{0}^{H}\right)^{2}=$ $16 d^{2} \beta^{2} u^{*}\left(\lambda_{0}^{H} \beta+u^{*}\right)>0, h(\lambda)=0$ has two positive roots as

$$
\begin{aligned}
& \underline{\lambda}=\frac{d}{\beta}\left(\lambda_{0}^{H} \beta+2 u^{*}-2 \sqrt{u^{*}\left(\lambda_{0}^{H} \beta+u^{*}\right)}\right), \\
& \bar{\lambda}=\frac{d}{\beta}\left(\lambda_{0}^{H} \beta+2 u^{*}+2 \sqrt{u^{*}\left(\lambda_{0}^{H} \beta+u^{*}\right)}\right) .
\end{aligned}
$$

By $h\left(d \lambda_{0}^{H}\right)=-4 d \lambda \beta u^{*}<0$, hence $\underline{\lambda}<d \lambda_{0}^{H}<\bar{\lambda}$. That is, when $\lambda \in\left(\underline{\lambda}, d \lambda_{0}^{H}\right), D_{n}(\lambda)>0$, for all $n \in N$. No steady state bifurcation occurs. Then the potential steady state bifurcation points reduce to the following set:

$$
\wedge_{2}=\{\lambda \in(0, \underline{\lambda}] \mid(35) \text { are satisfied }\},
$$

with $\alpha, \beta, l, d$ fixed.

Next, the properties of $P_{+}(\lambda), \lambda \in(0, \underline{\lambda}]$ are discussed and direct computation can obtain the following results.

Lemma 6. Suppose that $\alpha \beta>1+\beta, \alpha \beta^{2}+2 \beta>(\alpha \beta+1)^{2}$ hold. When $\lambda \in(0, \underline{\lambda}], P_{+}(\lambda)$ is decreasing on $\lambda$ and $P_{-}(\lambda)$ is increasing on $\lambda$. Furthermore,

$$
\begin{gathered}
\lim _{\lambda \rightarrow 0^{+}} P_{+}(\lambda)=\lambda_{0}^{H} \beta, \\
P_{+}\left(\underline{\lambda^{-}}\right)=P_{-}\left(\underline{\lambda^{-}}\right)=\frac{\left(d \lambda_{0}^{H}-\underline{\lambda}\right) \beta}{2 d}, \\
\lim _{\lambda \rightarrow 0^{+}} P_{-}(\lambda)=0, \quad \lim _{\lambda \rightarrow \underline{\lambda}^{-}} P_{+}^{\prime}(\lambda)=-\infty, \\
\lim _{\lambda \rightarrow \underline{\lambda}^{-}} P_{-}^{\prime}(\lambda)=+\infty .
\end{gathered}
$$

From the properties of $P_{ \pm}(\lambda)$, if $0<p=n^{2} / l^{2}<\lambda_{0}^{H} \beta$, then there exists a unique

$$
\lambda_{n}^{S}=\frac{p \beta d \lambda_{0}^{H}-d p^{2}}{p \beta+\beta u^{*}} \in(0, \underline{\lambda}],
$$

such that

$$
P_{+}\left(\lambda_{n}^{S}\right)=\frac{n^{2}}{l^{2}} \quad \text { or } \quad P_{-}\left(\lambda_{n}^{S}\right)=\frac{n^{2}}{l^{2}} .
$$

Thus, $D_{n}\left(\lambda_{n}^{S}\right)=0$.

Define $\tilde{l}_{n}=n / \sqrt{\lambda_{0}^{H} \beta}$. Then for any $l>\tilde{l}_{n}$, there exists a unique $\lambda_{i}^{S} \in(0, \underline{\lambda}]$ such that $i^{2} / l^{2}=P_{+}\left(\lambda_{i}^{S}\right)$ or $i^{2} / l^{2}=P_{-}\left(\lambda_{i}^{S}\right)$.

$\lambda_{n}^{S} \in(0, \underline{\lambda}]$ are potential steady state bifurcation values. But it is possible that for some $i, j \in N$ and $i \neq j$ such that $P_{+}\left(\lambda_{i}^{S}\right)=P_{-}\left(\lambda_{j}^{S}\right)$. In this case, 0 is a double eigenvalue of $L(\lambda)$ for $\lambda=\lambda_{i}^{S}=\lambda_{j}^{S}$. In addition, it is also possible that $\lambda_{i}^{S}=\lambda_{j}^{H}$. Hence, the dimension of center manifold at $E\left(u^{*}, v^{*}\right)$ can be as high as 3 .

Next we will prove that there are only countable many $l>0$, in fact only finitely many $l \in(0, M)$ for any given $M>0$, such that $\lambda_{i}^{S}=\lambda_{j}^{S}, \lambda_{i}^{S}=\lambda_{j}^{H}$ for some $i, j \in N$.
Let $E_{n}(\lambda, l)=l^{2} T_{n}(\lambda), F_{n}(\lambda, l)=l^{4} D_{n}(\lambda)$ be polynomials of $\lambda$ and $l$. Hence, on $(\lambda, l)$-plane, $q_{n}=\left\{(\lambda, l): E_{n}(\lambda, l)=0\right\}, p_{n}=$ $\left\{(\lambda, l): F_{n}(\lambda, l)=0\right\}$ are finitely many analytic curves. We fix $\lambda \in(0, \underline{\lambda}]$, so for any $M>0$, there are only finitely many $i, j \in$ $N$ such that $q_{i} \bigcap((0, \underline{\lambda}] \times[0, M]) \neq \emptyset, p_{j} \cap((0, \underline{\lambda}] \times[0, M]) \neq \emptyset$. So $p_{j}, q_{i}$ have at most only finitely many intersection points. Thus the intersection points of different $q_{i}, p_{j}$ in $(0, \underline{\lambda}] \times$ $[0,+\infty]$ are at most countable. Define

$$
\begin{aligned}
L^{E}= & \left\{l>0 \mid F_{i}(\lambda, l)=F_{j}(\lambda, l)\right. \\
& \text { or } E_{i}(\lambda, l)=F_{j}(\lambda, l)
\end{aligned}
$$

for some $\lambda \in(0, \underline{\lambda}]$, and $i, j \in N\}$.

We assume that $l \in R / L^{E}$, and thus 0 is a simple eigenvalue. And

$$
\frac{d D\left(\lambda_{n}^{S}\right)}{d \lambda}=p \beta+\beta u^{*}>0
$$

Obviously, $T_{m}\left(\lambda_{n}^{S}\right) \neq 0, D_{m}\left(\lambda_{n}^{S}\right) \neq 0$, for all $m \neq n$.

Summarizing the above, we can obtain the main results in this section.

Theorem 7. Suppose that $\alpha, \beta, d$ are positive constants and $\alpha \beta>1+\beta, \alpha \beta^{2}+2 \beta>(\alpha \beta+1)^{2}$ hold. $P_{ \pm}(\lambda)$ are defined as (38) and $\tilde{l}_{n}=n / \sqrt{\lambda_{0}^{H} \beta}$.

If for some $n \in N, l \in\left(\widetilde{l}_{n},+\infty\right) \backslash L^{E}$, there exactly exists one point $\lambda_{n}^{S}=\left(\beta d \lambda_{0}^{H} p-d p^{2}\right) /\left(\beta p+\beta u^{*}\right) \in(0, \underline{\lambda})$, such that $p_{+}\left(\lambda_{n}^{S}\right)=n^{2} / l^{2}$ or $P_{-}\left(\lambda_{n}^{S}\right)=n^{2} / l^{2}$, where $p=n^{2} / l^{2}$. Then there is a smooth curve $\Gamma_{n}$ of positive solutions of (33) bifurcating from $\left(\lambda_{n}^{S}, u^{*}, v^{*}\right)$. Moreover, near $\left(\lambda_{n}^{S}, u^{*}, v^{*}\right), \Gamma_{n}=$ $\{\lambda(s), u(s), v(s): s \in(-\epsilon,+\epsilon)\}$, where

$$
\begin{aligned}
& u(s)=u^{*}+s a_{n} \cos \frac{n x}{l}+s \psi_{1}(s), \\
& v(s)=v^{*}+s b_{n} \cos \frac{n x}{l}+s \psi_{2}(s),
\end{aligned}
$$

for $s \in(-\epsilon,+\epsilon)$ for some $C^{\infty}$ smooth function $\lambda, \psi_{1}, \psi_{2}$ such that $\lambda(0)=\lambda_{n}^{S}$ and $\psi_{1}(0)=\psi_{2}(0)=0$. Here $a_{n}, b_{n}$ satisfy $L_{n}\left(\lambda_{n}^{S}\right)\left(a_{n}, b_{n}\right)^{T}=(0,0)^{T}$.

In the following, we will discuss the interaction between the Hopf and steady state bifurcation points. All possible Hopf bifurcation points $\lambda_{i}^{H}$ are identified in Section 2.1 and all possible steady state bifurcation points $\lambda_{j}^{S}$ can be identified in Section 3. And it has previously shown that at most countable $l \in L^{E}$ such that $\lambda_{j}^{H}=\lambda_{i}^{S}$ or $\lambda_{i}^{S}=\lambda_{j}^{S}$ for some $i, j \in N$. Hence high-dimensional center manifold from $\left(\lambda, u^{*}, v^{*}\right)$ is still possible. We do not consider it here. We assume $l \notin L^{E}$, Hopf bifurcations, and steady state bifurcation points may occur at this points in the following four cases. 


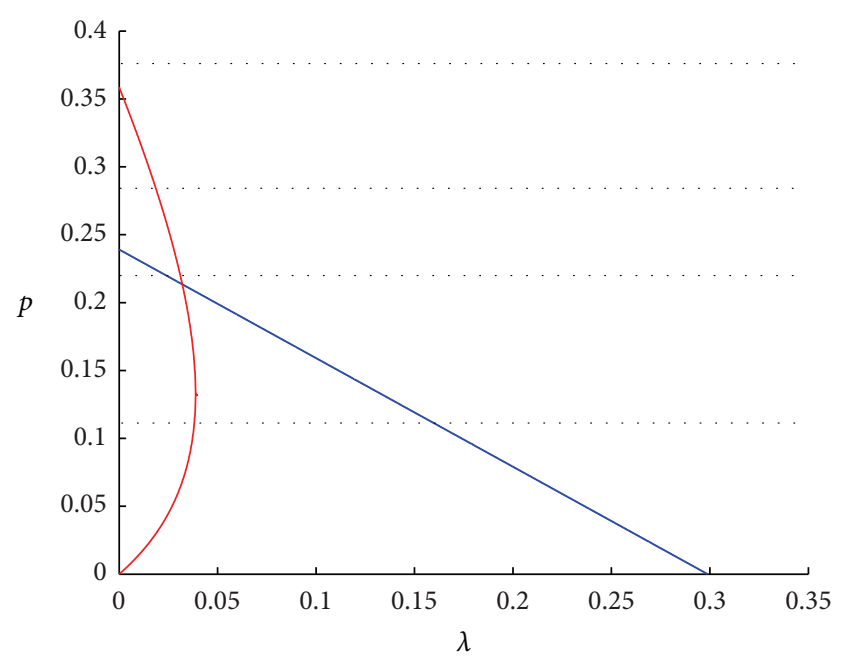

Figure 3: Graph of $E_{n}(\lambda, p) F_{n}(\lambda, p)=0$. Here $d=0.5, \alpha=0.4$, $\beta=1.2, l=30, u^{*}=0.1892, v^{*}=0.227$, and $\lambda_{0}^{H}=0.2989$. The horizontal lines are $p=n^{2} / l^{2}$, where $n=0,1,2, \ldots$

Case 1. Neither $\lambda_{i}^{H}$ nor $\lambda_{j}^{S}$ exists, and then there is no bifurcation points;

Case 2. $\lambda_{j}^{S}$ exists but not $\lambda_{i}^{H}$, and then there is one steady state bifurcation point and no Hopf bifurcation point.

Case 3. Both $\lambda_{j}^{S}$ and $\lambda_{i}^{H}$ exist, and then

(a) $\lambda_{i}^{H}<\lambda_{j}^{S}$; then there is one steady state bifurcation point and no Hopf bifurcation since $D_{i}\left(\lambda_{i}^{H}\right)<0$;

(b) $\lambda_{j}^{S}<\lambda_{i}^{H}$; then there is one steady state bifurcation point and one Hopf bifurcation.

Case 4. $\lambda_{i}^{H}$ exists but no $\lambda_{j}^{S}$, then there is no steady state bifurcation point and one Hopf bifurcation.

Example 8. In Figure 3, for $n \geq 18$ and $n \in N$, Case 1 occurs, and there are no bifurcation points. For $15 \leq n \leq 17$, Case 2 occurs, and there exists 3 steady state bifurcation points and no Hopf bifurcation points. For $n=14$, Case 3 (a) occurs, and there is one steady state bifurcation point $\lambda_{14}^{S}=0.0314$ and no Hopf bifurcation points since $D_{14}\left(\lambda_{14}^{H}\right)<0$. For $1 \leq$ $n \leq 13$, Case 3 (b) occurs, and there exists 13 Hopf bifurcation points and 13 steady state bifurcation points. For $n=0$, Case 4 occurs, and there exists one Hopf bifurcation point and no steady state bifurcation points.

\section{Global Stability of the Positive Coexistence}

In this section, we will prove the global stability of the positive constant coexistence by the upper and lower solutions method in Pao [23, 24] and Chen and Shi [13].

Theorem 9. Assume that $d, \beta, \lambda$ are positive constants and $\alpha>1$. Then for system (5), the positive constant equilibrium
$E\left(u^{*}, v^{*}\right)$ is globally asymptotically stable if $\alpha \beta<1$ or $\alpha \beta>$ $1+\beta$. That is, for any initial values $u_{0}(x)>0, v_{0}(x) \geq 0$,

$$
\begin{array}{r}
\lim _{t \rightarrow \infty} u(x, t)=u^{*}, \quad \lim _{t \rightarrow \infty} v(x, t)=v^{*}, \\
\text { uniformly in } x \in[0, l \pi]
\end{array}
$$

if

$$
\alpha \beta<1 \text { or } \alpha \beta>1+\beta .
$$

Proof. It is well known that if $c>0$ and $w(x, t)>0$ satisfies

$$
\begin{gathered}
\frac{\partial w}{\partial t}=D \Delta w+w(c-w), \quad x \in \Omega, t>0, \\
\frac{\partial w}{\partial \nu}=0, \quad x \in \partial \Omega, \\
w(x, 0) \geq(\not \equiv) 0, \quad x \in \Omega,
\end{gathered}
$$

then $\lim _{t \rightarrow \infty} w(x, t)=c$ uniformly in $x \in \bar{\Omega}$.

We can choose $\epsilon_{0}$ satisfying

$$
0<\epsilon_{0}<\frac{\beta(1-1 / \alpha)}{1+\beta}, \quad \text { since } \alpha>1 .
$$

By the first equation of (5), we have

$$
\frac{\partial u}{\partial t} \leq u_{x x}+u(x, t)(1-u(x, t)) .
$$

By the comparison principle of the parabolic equation, we obtain

$$
\limsup _{t \rightarrow+\infty} \max _{x \in[0, l \pi]} u(x, t) \leq 1
$$

Then there exists $t_{0}>0$ such that $u(x, t)<1+\epsilon_{0} \triangleq \bar{u}$, for all $t>t_{0}, x \in[0, l \pi]$. Thus, by the second equation of (5),

$$
\frac{\partial v}{\partial t} \leq d v_{x x}+\lambda v\left(\beta-\frac{v}{1+\epsilon_{0}}\right), \quad \forall t>t_{0} .
$$

By the comparison principle again, $\lim \sup _{t \rightarrow+\infty} \max _{x \in[0, l \pi]} v(x, t) \leq \beta\left(1+\epsilon_{0}\right)$. There exists $t_{1}>t_{0}$ such that $v(x, t)<\beta\left(1+\epsilon_{0}\right)+\epsilon_{0} \triangleq \bar{v}$, for all $t>t_{1}, x \in[0, l \pi]$.

On the other hand,

$$
\frac{\partial u}{\partial t}=u_{x x}+u\left(1-u-\frac{v}{u+\alpha v}\right) \geq u_{x x}+u\left(1-\frac{1}{\alpha}-u\right) .
$$

By the comparison principle, $\lim \inf _{t \rightarrow+\infty} \min _{x \in[0, l \pi]} u(x, t) \geq$ $1-1 / \alpha>0$, since $\alpha>1$. Thus, there exists $t_{2}>t_{1}$ such that $u(x, t)>1-1 / \alpha-\epsilon_{0} \triangleq \underline{u}>0$, for all $t>t_{2}$. Again in turn this implies

$$
\frac{\partial v}{\partial t} \geq d v_{x x}+\lambda v\left(\beta-\frac{v}{\underline{u}}\right), \quad t>t_{2} .
$$


Hence, $\lim \inf _{t \rightarrow+\infty} \min _{x \in[0, l \pi]} v(x, t) \geq \beta\left(1-1 / \alpha-\epsilon_{0}\right)$. There exists $t_{3}>t_{2}$ such that

$$
v(x, t)>\beta\left(1-\frac{1}{\alpha}-\epsilon_{0}\right)-\epsilon_{0} \triangleq \underline{v}>0, \quad \forall t>t_{3},
$$

since (51) holds. Therefore, for $t>t_{3}, \underline{u} \leq u(x, t) \leq \bar{u}, \underline{v} \leq$ $v(x, t) \leq \bar{v}$, for all $x \in[0, l \pi]$ and $\bar{u}, \underline{u}, \bar{v}, \underline{v}$ satisfy

$$
\begin{array}{ll}
1-\bar{u}-\frac{\underline{v}}{\bar{u}+\alpha \underline{v}}<0, & \beta-\frac{\bar{v}}{\bar{u}}<0, \\
1-\underline{u}-\frac{\bar{v}}{\underline{u}+\alpha \bar{v}}>0, & \beta-\frac{\underline{v}}{\underline{u}}>0 .
\end{array}
$$

Equation (58) implies that $(\bar{u}, \bar{v})$ and $(\underline{u}, \underline{v})$ are a pair of upper and lower solutions of system (5), respectively (see $[23,24])$. It is obvious that there exists $M>0$ such that for any $(\underline{u}, \underline{v}) \leq$ $\left(u_{1}, v_{1}\right),\left(u_{2}, v_{2}\right) \leq(\bar{u}, \bar{v})$,

$$
\begin{gathered}
\left|u_{1}\left(1-u_{1}\right)-\frac{u_{1} v_{1}}{u_{1}+\alpha v_{1}}-u_{2}\left(1-u_{2}\right)+\frac{u_{2} v_{2}}{u_{2}+\alpha v_{2}}\right| \\
\leq M\left(\left|u_{1}-u_{2}\right|+\left|v_{1}-v_{2}\right|\right), \\
\left|\lambda v_{1}\left(\beta-\frac{v_{1}}{u_{1}}\right)-\lambda v_{2}\left(\beta-\frac{v_{2}}{u_{2}}\right)\right| \leq M\left(\left|u_{1}-u_{2}\right|+\left|v_{1}-v_{2}\right|\right) .
\end{gathered}
$$

We construct the iteration sequences $\left(\bar{u}^{(m)}, \bar{v}^{(m)}\right)$ and $\left(\underline{u}^{(m)}, \underline{v}^{(m)}\right), m \geq 1$ satisfy

$$
\begin{aligned}
\bar{u}^{(m)}= & \bar{u}^{(m-1)}+\frac{1}{M} \bar{u}^{(m-1)} \\
& \times\left(1-\bar{u}^{(m-1)}-\frac{\underline{v}^{(m-1)}}{\bar{u}^{(m-1)}+\alpha \underline{v}^{(m-1)}}\right), \\
\bar{v}^{(m)}= & \bar{v}^{(m-1)}+\frac{1}{M} \lambda \bar{v}^{(m-1)}\left(\beta-\frac{\bar{v}(m-1)}{\bar{u}^{(m-1)}}\right), \\
\underline{u}^{(m)}= & \underline{u}^{(m-1)}+\frac{1}{M} \underline{u}^{(m-1)} \\
& \times\left(1-\underline{u}^{(m-1)}-\frac{\bar{v}^{(m-1)}}{\underline{u}^{(m-1)}+\alpha \bar{v}^{(m-1)}}\right), \\
\underline{v}^{(m)}= & \underline{v}^{(m-1)}+\frac{1}{M} \lambda \underline{v}^{(m-1)}\left(\beta-\frac{\underline{v}^{(m-1)}}{\underline{u}^{(m-1)}}\right),
\end{aligned}
$$

where $\left(\bar{u}^{(0)}, \bar{v}^{(0)}\right)=(\bar{u}, \bar{v}),\left(\underline{u}^{(0)}, \underline{v}^{(0)}\right)=(\underline{u}, \underline{v})$. Then by (58) we can obtain

$$
\begin{aligned}
(\underline{u}, \underline{v}) & \leq\left(\underline{u}^{(m)}, \underline{v}^{(m)}\right) \leq\left(\underline{u}^{(m+1)}, \underline{v}^{(m+1)}\right) \\
& \leq \cdots \leq\left(\bar{u}^{(m+1)}, \bar{v}^{(m+1)}\right) \\
& \leq\left(\bar{u}^{(m)}, \bar{v}^{(m)}\right) \leq(\bar{u}, \bar{v}) .
\end{aligned}
$$

Then there exist $(\widetilde{u}, \widetilde{v})$ and $(\underset{\sim}{u}, \underset{\sim}{v})$ such that

$$
\begin{array}{ll}
\lim _{m \rightarrow \infty} \bar{u}^{(m)}=\tilde{u}, & \lim _{m \rightarrow \infty} \bar{v}^{(m)}=\widetilde{v}, \\
\lim _{m \rightarrow \infty}{\underset{u}{\sim}}^{(m)}=\underset{\sim}{\mathcal{u}}, & \lim _{m \rightarrow \infty} \stackrel{v}{\sim}^{(m)}=\underline{\sim},
\end{array}
$$

and $(\underline{u}, \underline{v}) \leq(\underline{u}, \underset{v}{v}) \leq(\widetilde{u}, \widetilde{v}) \leq(\bar{u}, \bar{v})$. And

$$
\begin{aligned}
& 1-\tilde{u}-\frac{\stackrel{v}{\widetilde{u}+\alpha \underline{v}}}{\widetilde{u}}=0, \quad \beta-\frac{\widetilde{v}}{\widetilde{u}}=0, \\
& 1-\underset{\sim}{\underline{u}}-\frac{\widetilde{v}}{\underline{\sim}+\alpha \widetilde{v}}=0, \quad \beta-\frac{v}{\underline{u}}=0 .
\end{aligned}
$$

According to (63), we have

$$
\begin{aligned}
& (1-\tilde{u})(\tilde{u}+\alpha \beta \underline{u})=\beta \underset{\sim}{u}, \\
& (1-\underset{\sim}{u})(\underline{u}+\alpha \beta \tilde{u})=\beta \tilde{u} .
\end{aligned}
$$

By (64), we can obtain

$$
(\tilde{u}-\underset{\sim}{u})(1-\alpha \beta+\beta-\tilde{u}-\underset{\sim}{u})=0 .
$$

If we assume that $\tilde{u} \neq \underset{\sim}{u}$, then

$$
1-\alpha \beta+\beta-\tilde{u}-\underset{\sim}{u}=0 .
$$

Substituting (66) into (64) and simplifying, we can obtain

$$
\begin{aligned}
& (\alpha \beta-1) \tilde{u}^{2}+\tilde{u}\left(1-2 \alpha \beta+\alpha^{2} \beta^{2}-\alpha \beta^{2}+\beta\right) \\
& +\beta(\alpha-1)(1-\alpha \beta+\beta)=0, \\
& (\alpha \beta-1) \underset{\sim}{\mathcal{u}^{2}}+\underset{\sim}{u}\left(1-2 \alpha \beta+\alpha^{2} \beta^{2}-\alpha \beta^{2}+\beta\right) \\
& +\beta(\alpha-1)(1-\alpha \beta+\beta)=0 .
\end{aligned}
$$

Thus the quadratic equation

$$
\begin{gathered}
(\alpha \beta-1) x^{2}+x\left(1-2 \alpha \beta+\alpha^{2} \beta^{2}-\alpha \beta^{2}+\beta\right) \\
+\beta(\alpha-1)(1-\alpha \beta+\beta)=0
\end{gathered}
$$

has two different positive roots $\tilde{u}$ and $\underset{\sim}{u}$.

On the other hand, when $\alpha \beta<1$ or $\alpha \beta>1+\beta$,

$$
x_{1} x_{2}=\beta(\alpha-1) \frac{1-\alpha \beta+\beta}{\alpha \beta-1}<0,
$$

where $x_{1}, x_{2}$ are two roots of (68). Thus (68) cannot have two positive roots.

When $\alpha \beta=1$, (68) becomes a linear equation and has at most one real root. This contradicts the above. Hence, $\tilde{u}=\underset{\sim}{u}$. Similarly, $\widetilde{v}=\underset{v}{v}$. Then from the results in $[23,24]$, the solution $(u(x, t), v(x, t) \tilde{)}$ of System (5) satisfies

$$
\begin{array}{r}
\lim _{t \rightarrow+\infty} u(x, t)=u^{*}, \quad \lim _{t \rightarrow+\infty} v(x, t)=v^{*}, \\
\text { uniformly in } x \in[0, l \pi] .
\end{array}
$$

\section{Acknowledgments}

The author wish to express his special gratitude to the reviewers and the editor for their valuable comments and suggestions that led to a truly significant improvement of the paper. This research is partially supported by Shandong Provincial Natural Science Foundation, China (no. ZR2011AQ017), and by the Fundamental Research Funds for the Central Universities (nos. 13CX02011A and 12CX04081A). 


\section{References}

[1] G. Guo and J. Wu, "The effect of mutual interference between predators on a predator-prey model with diffusion," Journal of Mathematical Analysis and Applications, vol. 389, no. 1, pp. 179194, 2012.

[2] J. Tanner, "The stability and the intrinstic growth rates of prey and predator populations," Ecology, vol. 56, pp. 855-867, 1975.

[3] D. J. Wollkind, J. B. Collings, and J. A. Logan, "Metastability in a temperature-dependent model system for predator-prey mite outbreak interactions on fruit trees," Bulletin of Mathematical Biology, vol. 50, no. 4, pp. 379-409, 1988.

[4] E. Sáez and E. González-Olivares, "Dynamics of a predator-prey model," SIAM Journal on Applied Mathematics, vol. 59, no. 5, pp. 1867-1878, 1999.

[5] C. S. Holling, "The functional response of predator to prey density and its role in mimicry and population regulation," Memoirs of the Entomological Society of Canada, vol. 45, pp. 169, 1965.

[6] S. B. Hsu and T. W. Huang, "Global stability for a class of predator-prey systems," SIAM Journal on Applied Mathematics, vol. 55, no. 3, pp. 763-783, 1995.

[7] A. Gasull, R. Kooij, and J. Torregrosa, "Limit cycles in the Holling-Tanner model," Publicacions Matemàtiques, vol. 41, pp. 149-167, 1997.

[8] J. Shi and X. Wang, "On global bifurcation for quasilinear elliptic systems on bounded domains," Journal of Differential Equations, vol. 246, no. 7, pp. 2788-2812, 2009.

[9] F. Yi, J. Wei, and J. Shi, "Bifurcation and spatiotemporal patterns in a homogeneous diffusive predator-prey system," Journal of Differential Equations, vol. 246, no. 5, pp. 1944-1977, 2009.

[10] W. Zuo and J. Wei, "Multiple bifurcations and spatiotemporal patterns for a coupled two-cell Brusselator model," Dynamics of Partial Differential Equations, vol. 8, no. 4, pp. 363-384, 2011.

[11] Z.-P. Ma and W.-T. Li, "Bifurcation analysis on a diffusive Holling-Tanner predator-prey model," Applied Mathematical Modelling. Simulation and Computation for Engineering and Environmental Systems, vol. 37, no. 6, pp. 4371-4384, 2013.

[12] R. Peng and M. Wang, "Global stability of the equilibrium of a diffusive Holling-Tanner prey-predator model," Applied Mathematics Letters, vol. 20, no. 6, pp. 664-670, 2007.

[13] S. Chen and J. Shi, "Global stability in a diffusive Holling-Tanner predator-prey model," Applied Mathematics Letters, vol. 25, no. 3, pp. 614-618, 2012.

[14] R. Arditi and L. R. Ginzburg, "Coupling in predator-prey dynamics: ratio-dependence," Journal of Theoretical Biology, vol. 139, no. 3, pp. 311-326, 1989.

[15] R. Arditi, L. R. Ginzburg, and H. R. Akcakaya, "Variation in plankton densities among lakes: a case for ratio-dependent models," The American Naturalist, vol. 138, pp. 1287-1296, 1991.

[16] L. R. Ginzburg and H. R. Akcakaya, "Consequences of ratiodependent predation for steady-state properties of ecosystems," Ecology, vol. 73, no. 5, pp. 1536-1543, 1992.

[17] Q. Wang, Y. Zhang, Z. Wang, M. Ding, and H. Zhang, "Periodicity and attractivity of a ratio-dependent Leslie system with impulses," Journal of Mathematical Analysis and Applications, vol. 376, no. 1, pp. 212-220, 2011.

[18] Q. Wang, Jizhou, Z. Wang, M. Ding, and H. Zhang, "Existence and attractivity of a periodic solution for a ratio-dependent Leslie system with feedback controls," Nonlinear Analysis. Real World Applications, vol. 12, no. 1, pp. 24-33, 2011.
[19] M. Banerjee and S. Banerjee, "Turing instabilities and spatiotemporal chaos in ratio-dependent Holling-Tanner model," Mathematical Biosciences, vol. 236, no. 1, pp. 64-76, 2012.

[20] Z. Liang and H. Pan, "Qualitative analysis of a ratio-dependent Holling-Tanner model," Journal of Mathematical Analysis and Applications, vol. 334, no. 2, pp. 954-964, 2007.

[21] B. D. Hassard, N. D. Kazarinoff, and Y. H. Wan, Theory and Applications of Hopf Bifurcation, vol. 41, Cambridge University Press, Cambridge, UK, 1981.

[22] S. Ruan and J. Wei, "On the zeros of transcendental functions with applications to stability of delay differential equations with two delays," Dynamics of Continuous, Discrete \& Impulsive Systems A, vol. 10, no. 6, pp. 863-874, 2003.

[23] C. V. Pao, "On nonlinear reaction-diffusion systems," Journal of Mathematical Analysis and Applications, vol. 87, no. 1, pp. 165198, 1982.

[24] C. V. Pao, Nonlinear Parabolic and Elliptic Equations, Plenum Press, New York, NY, USA, 1992. 


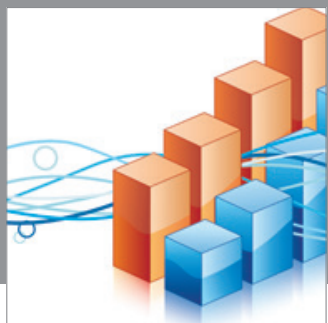

Advances in

Operations Research

mansans

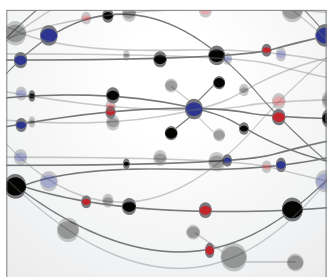

The Scientific World Journal
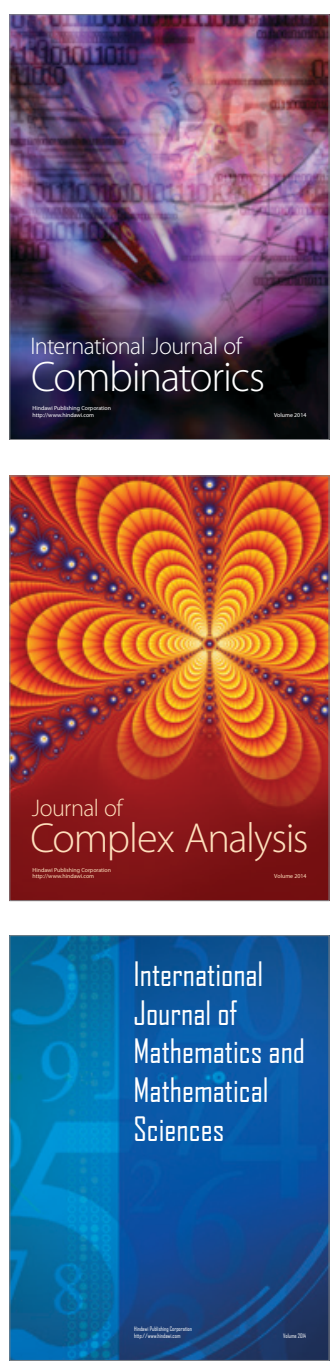
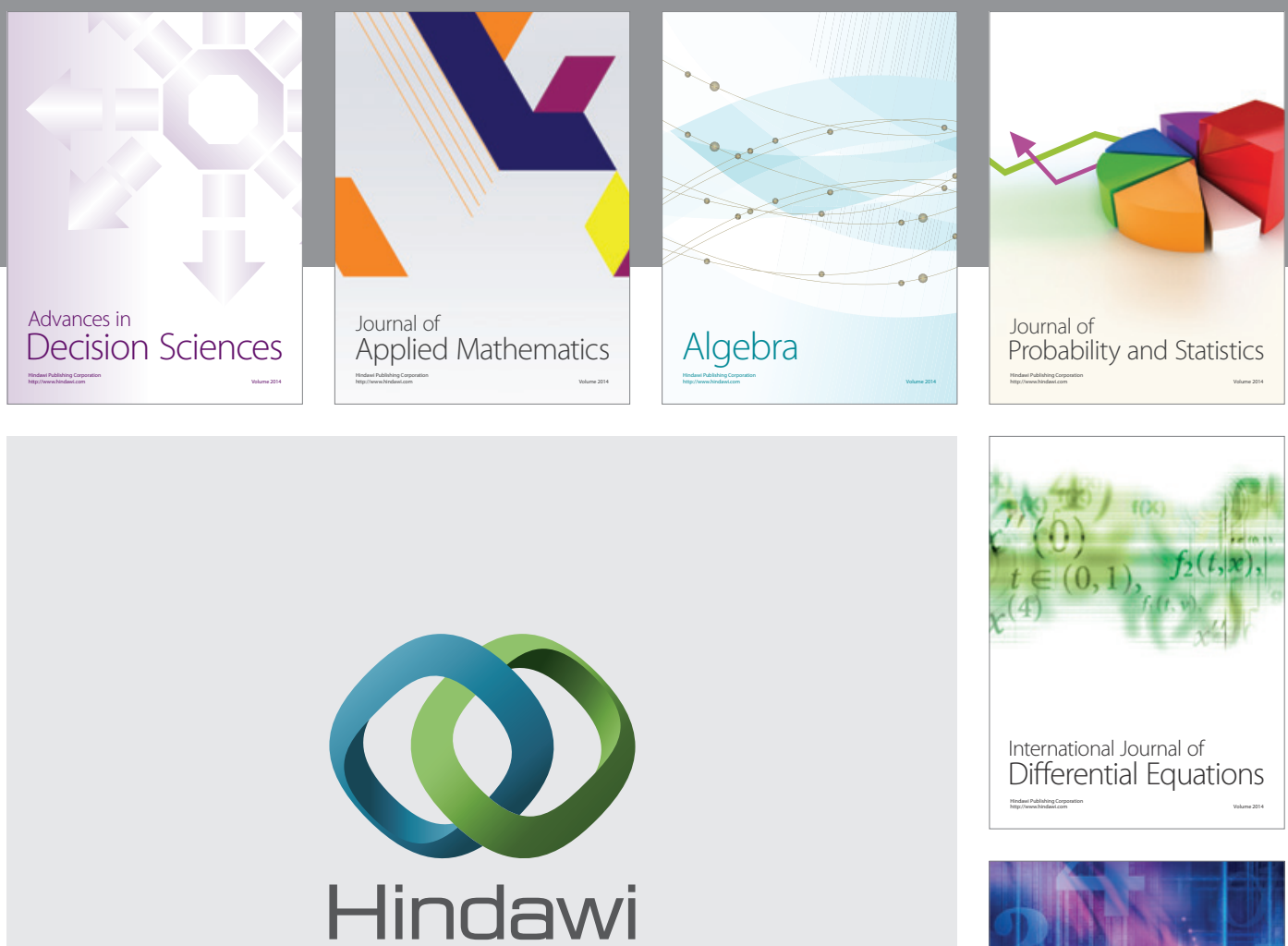

Submit your manuscripts at http://www.hindawi.com
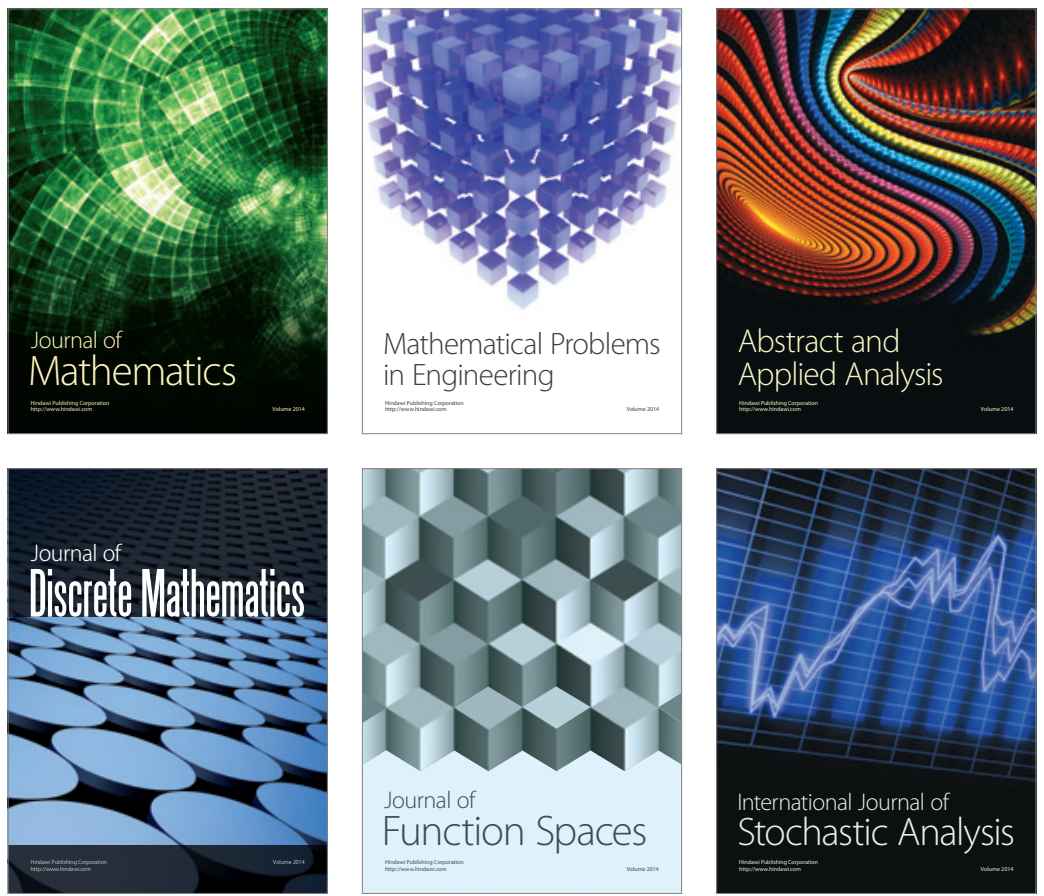

Journal of

Function Spaces

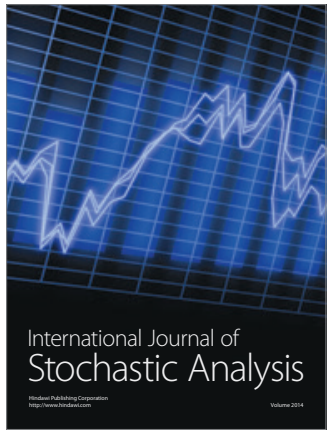

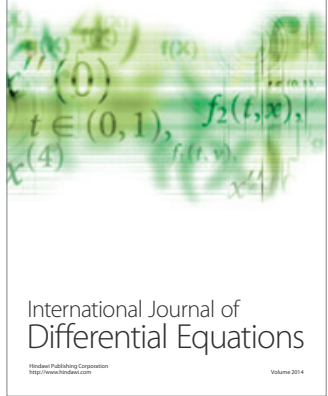
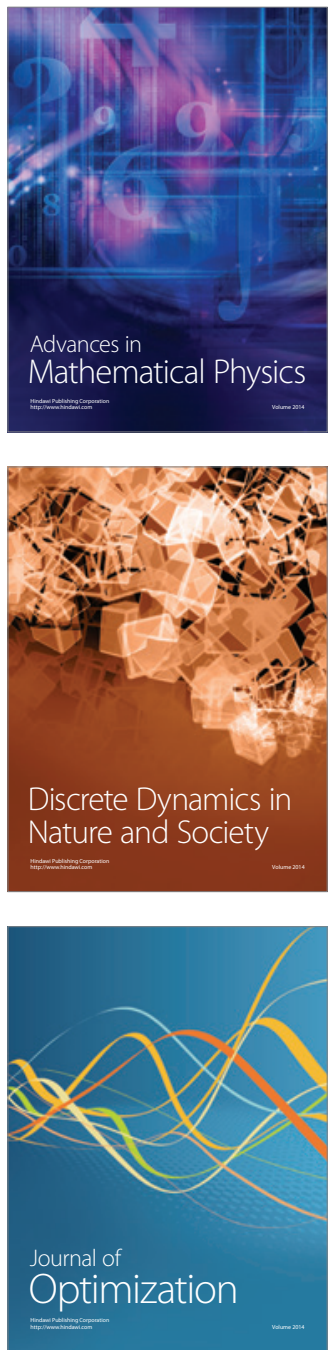